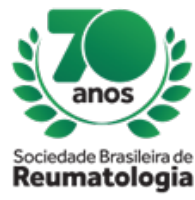

\title{
GRANULOMATOSIS WITH POLYANGIITIS SIMULATING RHEUMATOID ARTHRITIS: A CASE REPORT
}

Renan Rodrigues Neves Ribeiro Nascimento (Universidade Federal de São Paulo, São Paulo, SP, Brasil), Eduarda Bonelli Zarur (Universidade Federal de São Paulo, São Paulo, SP, Brasil), Daniel Viana da Silva Silva (Universidade Federal de São Paulo, São Paulo, SP, Brasil), Raquel Mitie Kanno (Universidade Federal de São Paulo, São Paulo, SP, Brasil), Igor Beltrão Duarte Fernandes (Universidade Federal de São Paulo, São Paulo, SP, Brasil), Germana Germana Ribeiro Araujo Carneiro Lucena (Universidade Federal de São Paulo, São Paulo, SP, Brasil), Mariana Davim Ferreira Gomes (Universidade Federal de São Paulo, São Paulo, SP, Brasil), Edgard Torres Reis Neto (Universidade Federal de São Paulo, São Paulo, SP, Brasil), Alexandre Wagner Silva Souza (Universidade Federal de São Paulo, São Paulo, SP, Brasil), Alexandre Lima Matos (Universidade Federal de São Paulo, São Paulo, SP, Brasil), Luíza Sá Rêgo Tupinambá (Universidade Federal de São Paulo, São Paulo, SP, Brasil)

\section{BACKGROUND}

Granulomatosis with polyangiitis (GPA) is a necrotizing vasculitis associated with antineutrophil cytoplasmic antibodies (ANCA) that affects predominantly small vessels. Although musculoskeletal involvement is found in a frequency of up to $49.15 \%$ in a series of cases, it is sometimes neglected in clinical practice. There is a paucity of literature regarding GPA manifesting as pseudo-rheumatoid arthritis and this case report exemplifies such association, so that it can be recognized and added to one of the differential diagnosis of Rheumatoid Arthritis (RA).

\section{CASE REPORT}

M.A.S, 63 years old female, from São Paulo, diagnosed with RA in 2008 due to polyarthritis of wrists, metacarpophalangeal and proximal interphalangeal joints, morning stiffness and rheumatoid factor (RF) of 1/320, without erosions (figure 1-2). In September 2017 it was decided to replace methotrexate with leflunomide due to sustained moderate disease activity. At that same time, she complained of weight loss, new onset non-secretive cough, unmeasured fever associated with sensory polyneuropathy in lower limbs. Chest tomography was performed and multiple cavitated lung nodules were found (figure 3). It was decided to suspend leflunomide due to the hypothesis of leflunomide induced pulmonary nodulosis as well as peripheral neurological disease secondary to the drug.

She was referred to the UNIFESP Rheumatology Department in 2018 and it was then decided to revisit the initial diagnosis due to the atypical RA presentation with sustained activity and no erosions associated with a severe pulmonary nodulosis not typically associated to leflunomide. New blood tests performed in 2018 showed negatives ACPA and ANA, RF of 1/320, and a positive p-ANCA, with negative anti-PR3 and Anti-MPO. An open lung biopsy was then performed, which showed a histopathological pattern of basophilic geographic necrosis, microabscesses, granulomatous reaction associated with multifocal vasculitis with negative culture, establishing the diagnosis of GPA. The patient is now being followed up in an outpatient clinic in the 4th cycle of cyclophosphamide with improvement of constitutional symptoms.

\section{CONCLUSION}

There are currently several medical conditions that mimic RA such as Gout, Sarcoidosis, Leprosy and Amyloidosis. However, although GPA presents with polyarthritis from small to large joints, sometimes deforming, it is often not remembered for its musculoskeletal involvement. Thus, we emphasize the importance of including GPA as a differential diagnosis of RA, especially when the presentation is atypical with constitutional, pulmonary and neurological symptoms as in this case reported. 